\section{A visionary pair}

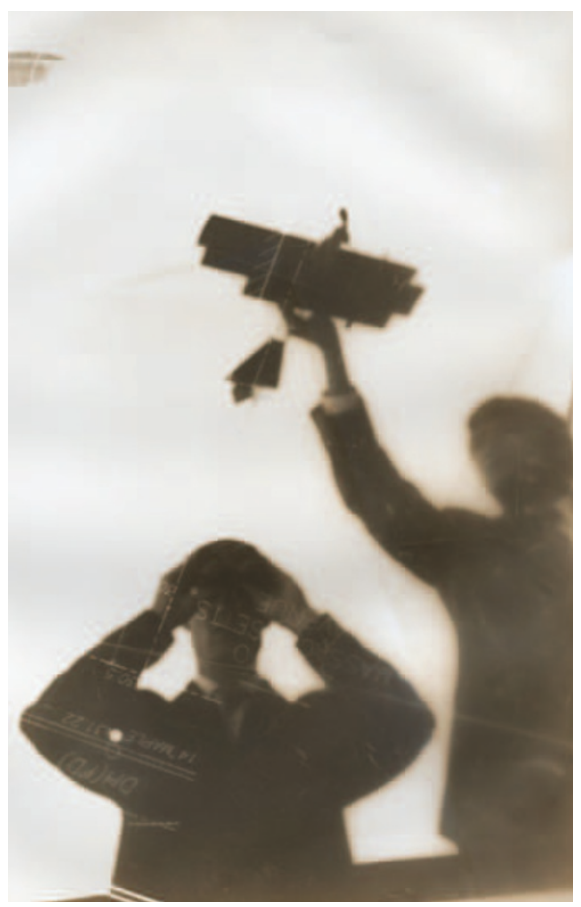

In a seminal paper in 1961, Jacob and Monod proposed a general model for bacterial gene regulation. Mainly on the basis of genetic information, they predicted the existence of repressors encoded by a new class of nonstructural genes. Repressors controlled the transcription of single or groups of genes. They were regulated by metabolites or other environmental factors, which could promote or antagonize their function. Repressors acted through operators that functioned in cis with the regulated genes (see Milestone 5); however, the precise nature of the repressors and how they interacted with operators was unclear.

The repressor model was sufficient to explain the current knowledge of regulated gene expression without a need to invoke activators (see Milestone 4), and accounted for systems as diverse as the lactose (lac) system of Escherichia coli and lambda phage genes. In the lysogenic state, lambda phage DNA is present in infected bacteria as a prophage with most phage genes not being expressed. Activation of gene expression and a switch to the lytic phase can be triggered by stimuli such as ultraviolet light. Similarly, expression of several genes - organized in a unit known as the lac operon - encoding enzymes that metabolize lactose is induced by external lactose or other $\beta$-galactosides, such as isopropyl- $\beta$-D-thiogalactoside (IPTG). The repressor model postulated that, in both instances, the respective inducers function by inhibiting the lambda and lac repressors that otherwise repress gene expression.

This visionary paper stimulated a quest for the isolation of repressors, which culminated in the isolation of the lac and the lambda repressors in 1966 and 1967, respectively. Using a mutant strain that was hypersensitive to IPTG, in which they assumed the repressor to bind more tightly to the inducer, Gilbert and Müller-Hill biochemically isolated cell fractions that bound labelled IPTG. They identified a protein of $\sim 15-20$ $\mathrm{kDa}$ as the lac repressor. Using phage mutants in which most genes were shut off and by inhibiting host protein synthesis, Ptashne purified differentially labelled proteins from bacteria infected with either wild-type phage or repressor mutants and isolated the lambda repressor as a protein of $\sim 30 \mathrm{kDa}$.

The biochemical isolation of repressors paved the way for the elucidation of their mechanism of action. Subsequent work showed that both the lambda and lac repres-

\section{MILESTONE 3}

\section{The $\mathrm{X}$ factor}

Although geneticists knew that mammalian females had two $\mathrm{X}$ chromosomes, whereas males had only one $\mathrm{X}$ and one $\mathrm{Y}$ chromosome, nobody had proposed a mechanism for equalizing the gene dosage until a seminal paper by Mary Lyon in 1961. She realized that if mice with only one $\mathrm{X}$ and no $\mathrm{Y}$ chromosome (XO mice) could develop as females and live, then one of the two $\mathrm{X}$ chromosomes in mammals might not be needed and could be inactivated during development, explaining the darkly staining bundle of sex chromatin seen only in female cells. Combining this with observations of mouse coat colour, she suggested that the inactivation of paternal versus maternal $\mathrm{X}$ was random in groups of cells, producing a mottled coat pattern when the colour gene was on the $\mathrm{X}$ chromosome. A similar pattern is seen in the tortoiseshell cat, as Lyon points out in her paper.
This revolutionary idea was supported by direct evidence from humans 2 years later. Ronald Davidson and colleagues confirmed, using gel electrophoresis, that some females had two forms of the enzyme glucose-6phosphate dehydrogenase (G6PD), encoded by alleles on the two $\mathrm{X}$ chromosomes. However, when individual cells were cloned and tested, each clone now only expressed one form or the other, not both. So, females clearly expressed only one G6PD gene in each cell, and the other appeared to be silenced. The authors cited data from contemporary papers showing that the entire second $\mathrm{X}$ might not be inactivated, which has now been confirmed by numerous direct surveys of gene activity.

Work on X-chromosome inactivation continued, and it was soon shown that the $\mathrm{X}$ inactivation signal seemed to emanate in cis from a centre in approximately the middle of the human chromosome, named the XIC. However, it was not until 1991 that Carolyn Brown et al. from the group of Huntington Willard characterized the first gene expressed exclusively from the inactive $\mathrm{X}$, specifically from the XIC. They could not find any evidence of protein made from this gene and therefore named it $\mathrm{X}$ (inactive)-specific transcript (XIST). When examined in cells with multiple inactive $\mathrm{X}$ chromosomes, the gene was expressed from all of the inactive $\mathrm{X}$ chromosomes, and certainly met the criteria for a cis-acting signal for inactivation. Their follow-up paper, a year later, confirmed that XIST was active as a large RNA molecule, not as a protein, and it could be seen to coat the inactive $\mathrm{X}$ chromosome in female cells.

These amazing papers helped open the way for the field of epigenetics and highlighted the need to think about more than DNA to truly understand the conversion of genotype to phenotype. Intensive studies now focus on how the XIST RNA comes to coat the soonto-be inactive $\mathrm{X}$ and interacts with recently described components of the chromatin machinery to effectively silence almost an entire chromosome.

Chris Gunter, Senior Editor, Nature

References and links

ORIGINAL RESEARCH PAPERS Lyon, M. F. Gene action in the X-chromosome of the mouse (Mus musculus L.). Nature 190, 372-373 (1961) | Davidson, R. G., Nitowsky, H. M. \& Childs, B. Demonstration of two populations of cells in the human female heterozygous for glucose-6-phosphate dehydrogenase variants. Proc. Natl Acad. Sci. USA 50 481-485 (1963) | Brown, C. J. et al. A gene from the region of the human $\mathrm{X}$ inactivation centre is expressed exclusively from the inactive X chromosome. Nature 349, 38-44 (1991) FURTHER READING Lyon, M. F. Sex chromatin and gene 